\title{
Laboratory-induced learned helplessness attenuates approach motivation as indexed by posterior versus frontal theta activity
}

\author{
Samantha J. Reznik ${ }^{1}$ Robin Nusslock ${ }^{1}$ Narun Pornpattananangkul ${ }^{1,2}$. \\ Lyn Y. Abramson ${ }^{3}$. James A. Coan ${ }^{4}$ • Eddie Harmon-Jones ${ }^{5}$
}

Published online: 5 June 2017

(C) Psychonomic Society, Inc. 2017

\begin{abstract}
Research suggests that midline posterior versus frontal electroencephalographic (EEG) theta activity (PFTA) may reflect a novel neurophysiological index of approach motivation. Elevated PFTA has been associated with approachrelated tendencies both at rest and during laboratory tasks designed to enhance approach motivation. PFTA is sensitive to changes in dopamine signaling within the fronto-striatal neural circuit, which is centrally involved in approach motivation, reward processing, and goal-directed behavior. To date, however, no studies have examined PFTA during a laboratory task designed to reduce approach motivation or goaldirected behavior. Considerable animal and human research supports the hypothesis put forth by the learned helplessness theory that exposure to uncontrollable aversive stimuli decreases approach motivation by inducing a state of perceived uncontrollability. Accordingly, the present study examined the effect of perceived uncontrollability (i.e., learned helplessness) on PFTA. EEG data were collected from 74 participants (mean age $=19.21$ years; 40 females) exposed to either Controllable $(n=26)$ or Uncontrollable $(n=25)$ aversive
\end{abstract}

Robin Nusslock

nusslock@northwestern.edu

1 Department of Psychology, Northwestern University, 2029 Sheridan Road, Evanston, IL 60208, USA

2 Department of Psychology, National University of Singapore, Singapore, Singapore

3 Department of Psychology, University of Wisconsin-Madison, Madison, WI, USA

4 Department of Psychology, University of Virginia, Charlottesville, VA, USA

5 School of Psychology, The University of New South Wales, Sydney, NSW, USA noise bursts, or a No-Noise Condition $(\mathrm{n}=23)$. In line with prediction, individuals exposed to uncontrollable aversive noise bursts displayed a significant decrease in PFTA, reflecting reduced approach motivation, relative to both individuals exposed to controllable noise bursts or the No-Noise Condition. There was no relationship between perceived uncontrollability and frontal EEG alpha asymmetry, another commonly used neurophysiological index of approach motivation. Results have implications for understanding the neurophysiology of approach motivation and establishing PFTA as a neurophysiological index of approach-related tendencies.

Keywords Posterior versus frontal theta activity $\cdot$ Approach motivation $\cdot$ Perceived uncontrollability $\cdot$ Learned helplessness $\cdot$ Depression

Identifying neurophysiological indices of approach motivation can inform our understanding of the mechanisms underlying affective or motivational tendencies and help establish endophenotypic markers of psychiatric disorders characterized by abnormal approach motivation and goal-directed activity (e.g., depression) (Gottesman \& Gould, 2003). Growing evidence suggests that midline posterior versus frontal electroencephalographic (EEG) theta activity (PFTA) may reflect a novel neurophysiological index of approach motivation. PFTA constitutes a difference score between posterior $(\mathrm{Pz})$ and frontal (Fz) midline theta activity (i.e., Pz-Fz; 4-8 Hz). PFTA has been shown to reflect approach motivational tendencies both at rest (Wacker, Chavanon, \& Stemmler, 2010) and during laboratory tasks designed to enhance approach motivation and goal-directed behavior (Walden, Pornpattananangkul, Curlee, Pornpattananangkul, \& Nusslock, 2015). PFTA is sensitive to changes in dopamine (Chavanon, Wacker, \& Stemmler, 2011; Wacker, Chavanon, 
\& Stemmler, 2006), the neurotransmitter most centrally involved in reward processing and goal-directed behavior (Berridge, 2007; Berridge, Robinson, \& Aldridge, 2009; Haber \& Knutson, 2010; Kringelbach \& Berridge, 2010; Schultz, 2002). Despite the promise of PFTA as a neurophysiological indicator of approach motivation, research has yet to examine PFTA during a laboratory task designed to reduce or attenuate approach-related motivation. Examining this question is critical to determining whether PFTA reflects a neurophysiological index of both elevated and attenuated approach motivational tendencies. Accordingly, the present study examined the effect of perceived uncontrollability on PFTA given considerable animal and human research indicating that exposure to uncontrollable aversive stimuli (i.e., learned helplessness) is associated with reduced approach motivation and decreased goal-directed behaviors. Analyses also examined the effect of perceived uncontrollability on frontal EEG alpha asymmetry, another frequently used neurophysiological index of approach motivation.

\section{Approach motivation and posterior versus frontal theta activity}

Although many regions in the brain facilitate goal-directed behavior, the fronto-striatal neural circuit is at the heart of the approach-motivational system (Berridge, 2007; Berridge et al., 2009; Haber \& Knutson, 2010; Kringelbach \& Berridge, 2010; Schultz, 2002). This circuit involves dopaminergic projections from midbrain nuclei (e.g., the ventral tegmental area) to subcortical regions that are central to processing the rewarding properties of stimuli and facilitating goaldirected behavior (e.g., the ventral striatum) to cortical target regions. Research has also implicated the rostral anterior cingulate cortex (rACC) in reward processing and approach motivation (Allman, Hakeem, Erwin, Nimchinsky, \& Hof, 2001; Baker \& Holroyd, 2011; Hayden, Pearson, \& Platt, 2009). The rACC receives concentrated dopaminergic inputs (Huang et al., 2013; Schweimer \& Hauber, 2006), shows increased activity in response to dopamine agonists (Udo de Haes, Maguire, Jager, Paans, \& Boer, 2007), and is modulated by genes governing dopamine transmission (Blasi et al., 2005). The rACC has also been implicated in depressive anhedonia, a symptom characterized by a loss of interest and reduced approach motivation (Wacker, Dillon, \& Pizzagalli, 2009).

There is growing evidence that PFTA indexes approachrelated motivation and that it is modulated by dopaminerelated activity in the rACC. First, Wacker and colleagues (2010) reported that elevated PFTA at rest was associated with elevated self-reported behavioral approach system sensitivity (BAS), as measured by Carver and White's (1994) BAS scale, a commonly used index of approach motivation (Chavanon et al., 2011; Koehler et al., 2011; Wacker et al., 2010; Wacker
\& Gatt, 2010). Second, theta current density in the rACC accounts for a significant portion of the variance in PFTA (Chavanon et al., 2011). Third, profiles of resting delta/theta current in the rACC have been associated with both anhedonia and blunted ventral striatal activation in response to monetary rewards, as indexed by functional magnetic resonance imaging (fMRI) (Wacker et al., 2009). Finally, pharmacological agents that affect dopaminergic activity, such as the dopamine antagonist sulpiride, which is designed to attenuate dopaminerelated activity, have been shown to modulate PFTA (Chavanon, Wacker, \& Stemmler, 2013). For example, whereas unmedicated participants displayed a positive relationship between self-reported BAS sensitivity and PFTA, individuals who took a dopamine antagonist exhibited a negative relationship between BAS sensitivity and PFTA (Wacker et al., 2006). In addition to using pharmacological agents, studies also show different profiles of PFTA among people with different genetic polymorphisms related to dopaminergic levels, including DRD2 (Koehler et al., 2011) and COMT (Wacker \& Gatt, 2010). Furthermore, extroverts displayed increased PFTA and introverts displayed decreased PFTA during a working memory task, but this relationship was reversed among extroverts and introverts who had taken a dopamine antagonist (Wacker et al., 2006). The linkage of PFTA to dopamine signaling in the rACC gives it a critical advantage over other neurophysiological markers of approach motivation (e.g., frontal EEG alpha asymmetry) that have not typically been associated with activity in this circuit. The fronto-striatal neural circuit is the hub of reward processing in the brain (Berridge, 2007; Berridge et al., 2009; Haber \& Knutson, 2010; Kringelbach \& Berridge, 2010; Schultz, 2002) and the association of PFTA with dopaminergic activity in this circuit (specifically the rACC) validates it as indexing critical reward-related neural processes.

The majority of research on the relationship between PFTA and approach motivation has relied on resting indices of PFTA designed to assess more trait-like variability. Coan and colleagues' (2006) capability model, however, suggests that approach tendencies are more pronounced and more resistant to measurement error when elicited using emotionally salient tasks. One recent study examined PFTA during an ecologically valid autobiographical memory task designed to increase approach motivation (Walden et al., 2015). In line with prediction, PFTA was elevated during goal-striving autobiographical memories as well as anxious apprehension autobiographical memories characterized by approach-related tendencies. To date, however, no studies have assessed PFTA during a laboratory task designed to reduce or attenuate approachrelated motivation. For PFTA to serve as a neurophysiological index of approach motivation, it should be sensitive to both elevated and attenuated approach motivational tendencies. Thus, examining PFTA during a reduced approach motivational state has important implications for establishing PFTA 
as a neurophysiological index of the full spectrum of approach-related tendencies.

\section{Learned helplessness attenuates approach motivation}

Over 40 years ago, the Learned Helplessness Theory of depression proposed that perceived uncontrollability over aversive stimuli is associated with decreased approach motivation and reduced goal-directed activity (Abramson et al., 2002; Abramson, Metalsky, \& Alloy, 1989; Abramson, Seligman, \& Teasdale, 1978; Alloy, Peterson, Abramson, \& Seligman, 1984). In the extreme, this reduction in approach motivation is a proximal cause of depressive symptoms characterized by reduced approach motivation, such as anhedonia, decreased energy, and psychomotor retardation. Perceived uncontrollability is the perception that one does not have control over the occurrence or impact of a negative or aversive event/stimulus (Thompson, 1981). Learned helplessness, from this perspective, is an induced state in which one comes to expect that one's responses and the termination of an aversive event are independent of each other. This expectation typically results in decreased approach motivation and behavioral disengagement, as the individual no longer searches for solutions to the perceived problem or attempts to exert control over his or her circumstances (Abramson et al., 2002, 1978).

Consistent with the Learned Helplessness Theory, there is considerable evidence that perceived uncontrollability over aversive stimuli decreases approach motivation and goaldirected behavior. Animals exposed to uncontrollable aversive stimuli experience deficits in learning and motivation as well as increased stress responses compared to animals exposed to similar amounts of controllable aversive stimuli (Abramson et al., 1978, 2002; Grahn, Watkins, \& Maier, 2000). In addition to other neurotransmitters (e.g., serotonin), animal research implicates dopamine in perceived uncontrollability, as stressor controllability has been shown to modulate stressinduced dopamine efflux in the medial prefrontal cortex (Bland et al., 2003).

An important advantage of the Learned Helplessness Theory is that it has a corresponding laboratory task for human research, the Learned Helplessness Paradigm (Alloy et al., 1984; Hiroto \& Seligman, 1975). This paradigm typically includes three between-subjects conditions: controllable aversive events, uncontrollable aversive events, and a control condition (typically non-exposure to the aversive event). Using this paradigm, and consistent with the Learned Helplessness Theory, researchers have reported that humans exposed to uncontrollable events in the laboratory display cognitive deficits and reduced approach motivation on subsequent tasks compared to those exposed to controllable events (Alloy, Peterson, Abramson, \& Seligman, 1984; Hiroto \&
Seligman, 1975). For example, Hiroto and Seligman (1975) reported that individuals exposed to uncontrollable loud noise bursts evidenced greater cognitive-motivational deficits on a subsequent anagram task, taking longer to solve the anagrams and solving fewer anagrams correctly. Furthermore, perceived uncontrollability over stressful life events has been linked to disease and elevated depression (Ledrich \& Gana, 2013; Mineka \& Hendersen, 1985; Sanjuán \& Magallares, 2009). By contrast, perceived control over a stressor has been associated with reduced depression and disease (Mineka \& Henderson, 1985) and elevated approach motivation and enhanced reward-related brain function (Salomons, Nusslock, Detloff, Johnstone, \& Davidson, 2015).

\section{Present study}

The present study examined the effect of perceived uncontrollability over an aversive stimulus on PFTA. Participants completed the well-established Learned Helplessness Task (Alloy et al., 1984; Hiroto \& Seligman, 1975; Miller \& Seligman, 1975) while EEG data were collected. Importantly, this study is the first to examine EEG activity during the Learned Helplessness Task. As noted, this task is designed to induce a state of perceived uncontrollability over aversive stimuli. During this task, participants were randomly assigned to either controllable aversive noise bursts, uncontrollable aversive noise bursts, or a no-noise control condition. Given the effect of uncontrollable aversive stimuli on reducing approach motivation and goal-directed activity (Abramson et al., 1978; Alloy et al., 1984; Hiroto \& Seligman, 1975; Miller \& Seligman, 1975; Salomons et al., 2015), we predicted that participants exposed to uncontrollable aversive noise bursts would display significantly reduced PFTA compared to participants exposed to both controllable noise bursts and nonoise. Results in line with this prediction would have important implications for understanding the neurophysiology of approach motivation and establishing PFTA as a neurophysiological index of approach-related tendencies.

We also examined the effect of the Learned Helplessness Task on asymmetrical activity in the alpha frequency band (8$13 \mathrm{~Hz}$ ) over the frontal cortex. Frontal EEG alpha asymmetry is a frequently used neurophysiological index of approach motivation (see Coan \& Allen, 2004 for review). Increased relative left-frontal electroencephalographic (EEG) activity has been associated with a propensity to approach or engage a stimulus, whereas decreased relative left-frontal EEG activity indicates a propensity toward reduced approach-related affect or increased withdrawal motivation (see Allen \& Reznik, 2015; Coan \& Allen, 2004; Davidson, 1998; Harmon-Jones, Gable, \& Peterson, 2010; Nusslock, Walden, \& Harmon-Jones, 2015 for review). We predicted that, like PFTA, participants exposed to uncontrollable aversive noise 
bursts would display decreased relative left-frontal EEG activity, reflecting reduced approach motivation secondary to perceived uncontrollability over the aversive noise stimuli.

\section{Method}

\section{Participants}

Seventy-four participants (mean age $=19.21$ years; 40 females) completed the study. Participants were recruited from the Introduction to Psychology extra credit participant pool at the University of Wisconsin-Madison. Participants were righthanded $(<18$, Chapman Handedness Scale [Chapman \& Chapman, 1987]), native English speakers, and were not taking psychotropic medication at the time of the study. Participants provided informed consent before participation, and the University of Wisconsin-Madison Institutional Review Board approved the protocol.

\section{Procedure}

Participants were instructed that the experiment involved examining brain responses during an "information-processing task." Following consent and EEG cap application, participants completed resting baseline EEG recordings. Resting EEG data were recorded while participants sat quietly in a sound-attenuated room for an 8-min period consisting of a counterbalanced sequence of minute-long eyes-open and eyes-closed segments, as in previous research (HarmonJones, 2004; Tomarken, Davidson, Wheeler, \& Kinney, 1992). Resting EEG data were examined to confirm there were no systematic differences in EEG activity between groups at rest and to examine the effect of laboratoryinduced learned helplessness after adjusting for individual differences in resting brain activity. Participants next completed the Learned Helplessness Task during which EEG data were collected to assess the effect of laboratory-induced learned helplessness on neurophysiological activity.

Learned Helplessness Task Considerable evidence indicates that the Learned Helplessness Task is effective in reducing approach motivation and decreasing goal-directed behavior in humans by generating perceived uncontrollability over aversive stimuli (Alloy et al., 1984; Hiroto \& Seligman, 1975; Miller \& Seligman, 1975). Participants were randomly assigned to one of three conditions: (1) uncontrollable aversive noise bursts $(\mathrm{N}=25),(2)$ controllable aversive noise bursts $(\mathrm{N}=26)$, and (3) a no noise control condition $(\mathrm{N}=23)$. All three conditions consisted of 50 trials, with each trial lasting an average of $26 \mathrm{~s}$. Participants were given a small box with two spring-loaded buttons on the surface. Participants in the Uncontrollable and Controllable Conditions were exposed to a 90-db burst of noise on each trial lasting $5 \mathrm{~s}$ unless terminated. The noise bursts were presented to participants through Califone headphones with a 600-ohm impedance. Participants in both the Uncontrollable and Controllable Conditions were told that they could use the button box to stop each noise burst, and that it was their task to figure out the pattern of button presses that would turn off the noise once it started. However, only participants in the Controllable Condition were able to turn off the noise by identifying a particular response pattern on the button box (pressing the right button two times). All participants in the Controllable Condition solved the response pattern and gained control over the duration of the noise bursts at some point during the task.

By contrast, participants in the Uncontrollable Condition had no control over when the noise was terminated. Importantly, the duration of each noise burst in the Uncontrollable Condition was yoked participant-byparticipant to individuals in the Controllable Condition. As such, a given participant in the Uncontrollable Condition received the same duration of each noise burst as the participant to whom they were yoked in the Controllable Condition. Yoking in this manner ensured that individuals in both conditions received the identical number, duration, and patterning of noise bursts. Participants in both the Controllable and the Uncontrollable Conditions received visual feedback for each trial on a computer monitor as to whether their own response terminated the noise (Green Square $=$ participant's response terminated the noise; Red Square = participant's response did not terminate the noise). For participants in the Controllable Condition, feedback was based on their actual response, whereas participants in the Uncontrollable Condition received a Red Square for each trial regardless of response. This design prevented participants in the Uncontrollable Condition from artificially perceiving control as the length of the noise presentations in the Uncontrollable Condition varied as a function of the performance of participants in the Controllable Condition given our yoking strategy.

Each trial began with a $7 \mathrm{~s}$ fixation cross that appeared on the computer monitor before the onset of the noise burst. This $7 \mathrm{~s}$ period was the EEG epoch of primary interest for each trial given Davidson's (1994) suggestion that approach motivation is most reliably modulated by cues during the foreperiod of a trial.

Participants in the No-Noise Condition engaged in a simple 50-trial task and were not exposed to any noise bursts. This task involved viewing red and green-colored squares (one square per trial). Using the button box, participants in this condition were instructed to push the right button twice in response to the Red Square, and the left button twice in response to the Green Square. For each trial there was a $7 \mathrm{~s}$ fixation cross that appeared on the computer monitor before the onset of the colored square. This $7 \mathrm{~s}$ EEG epoch provided a comparable period for each trial across the three conditions 
Controllable, Uncontrollable, and No-Noise. The total duration of the No-Noise Condition was equivalent to the Controllable and Uncontrollable Conditions. The No-Noise Condition served as a baseline assessment of EEG activity to facilitate the comparison of EEG activity between the Controllable and Uncontrollable Conditions.

\section{EEG recording and reduction}

Resting EEG recordings involved eight $60 \mathrm{~s}$ recordings consisting of a counterbalanced sequence of eyes-open and eyes-closed segments. EEG data for the uncontrollability task were epoched for each trial during the seven second fixation period preceding the onset of the noise burst for the Uncontrollable and Controllable Conditions, or preceding the onset of the colored square for the No-Noise Condition. Data were collected from 16 electrodes (CZ, PZ, FP1/FP2, F3/ $\mathrm{F} 4, \mathrm{~F} 7 / \mathrm{F} 8, \mathrm{C} 3 / \mathrm{C} 4, \mathrm{~T} 3 / \mathrm{T} 4, \mathrm{~T} 5 / \mathrm{T} 6$, and P3/P4), grounded at AFZ, and attached to a stretch-lycra electrode cap (Electrocap, Eaton, OH, USA). The online reference was the left earlobe (A1), and data were recorded from the right ear (A2), enabling computation of an offline average ears' reference (impedances $<5 \mathrm{k} \Omega$; homologs $\pm 1 \mathrm{k} \Omega$ ). Data were filtered (0.1-100 Hz; 60-Hz notch filter enabled), amplified, and digitized $(500 \mathrm{~Hz})$.

The EEG and electro-oculogram signals were visually scored and portions of the data containing aberrant eye movements, muscle movements, or other sources of artifact were removed. When artifact occurred in one channel at a point in time, data from all channels were removed. A vertical electrooculogram (VEOG) was then used in a regression-based artifact correction of the EEG as implemented in NeuroScan EDIT 4.5 (Neuroscan Inc.) based on a paper by Semlitsch and colleagues (1986). Specifically, part of the VEOG ( $\alpha$ -VEOG) was subtracted from EEG-activity: "corrected" $\mathrm{EEG}=$ "raw" EEG $-\alpha \cdot \mathrm{VEOG}$ (Semlitsch, Anderer, Schuster, $\&$ Presslich, 1986). Here $\alpha$ is determined by a regression analysis such that the covariance of VEOG and "corrected" EEG approach zero, theoretically: $\alpha=$ $\operatorname{cov}(V E O G$, "raw" EEG)/var(VEOG). Another visual inspection ensured that no aberrations remained. We only used the regression-based artifact correction for removing clearly defined eyeblinks. All non-blink horizontal and vertical eye movement, as well as data containing aberrant muscle movement, were manually removed based on visual inspection of the data. Derived averaged-ears reference data were used for further data reduction. Artifact-free epochs that were $1.024 \mathrm{~s}$ in duration for the Learned Helplessness Task and $2.048 \mathrm{~s}$ in duration for resting data were extracted through a Hamming window, which was used to prevent spurious estimates of spectral power. Epochs were overlapped by $75 \%$ for the Learned Helplessness Task and 50\% for the resting data to minimize loss of data due to Hamming window extraction.
A fast Fourier transform was used to calculate the power spectra.

In line with previous PFTA research (Wacker et al., 2009; Walden et al., 2015), total power in the theta band (4-8 Hz) was extracted from data collected both at rest and during the Learned Helplessness Task. Theta power was log-transformed and, as in previous research (Wacker et al., 2006, 2009; Walden et al., 2015), a midline posterior versus anterior theta difference score was computed $[\ln (\mathrm{Pz})-\ln (\mathrm{Fz})]$, with higher scores reflecting relatively greater posterior theta activity. As discussed, elevated posterior theta activity has been linked to greater approach motivation (Wacker et al., 2006, 2010).

In line with previous frontal EEG alpha asymmetry research (Allen, Coan, \& Nazarian, 2004; Coan \& Allen, 2004), total power in the alpha band $(8-13 \mathrm{~Hz})$ was extracted from data collected both at rest and during the Learned Helplessness Task. Greater alpha power at a given scalp electrode reflects less cortical activity (Allen et al., 2004). Alpha power was log-transformed and, as in previous research (Nusslock et al., 2011), we averaged alpha power in F3/F7 into a "left frontal region" and F4/F8 into a "right frontal region" and computed a composite frontal asymmetry index as follows: $[\ln ($ mean of F4/F8)- $\ln ($ mean of F3/F7) ]. We used a composite frontal symmetry index (a) because we did not have separate hypotheses for mid- (F3/F4) and lateral-frontal (F7/F8) electrodes, (b) because of the high correlation between mid- and lateral-frontal regions $(r s>.97)$, and (c) to minimize Type I error by reducing the number of statistical analyses.

\section{Data analysis}

As a manipulation check, we asked participants in the Controllable and Uncontrollable Conditions two questions on a 5-point scale at the completion of the study. First, we asked how much control they felt they had over the duration of the noise bursts during the Learned Helplessness Task ( $5=$ "I had complete control"; 1 = "I had no control"). Second, we asked how motivated they were to find the response pattern that turned off the noise bursts during the Learned Helplessness Task ( 5 = "Very motivated"; $1=$ "Not Motivated"). This latter question assessed whether the Learned Helplessness Task successfully reduced approach motivation and goal-directed behavior. T-tests compared participants in the Uncontrollable and Controllable Conditions on these measures of perceived controllability and approach motivation. The No-Noise Condition was not included in these analyses because there was no expectation of perceived uncontrollability or motivational deficits among these participants during the Learned Helplessness Task.

We first examined resting levels of both PFTA and frontal EEG alpha asymmetry to confirm that there were no differences in resting neurophysiological activity between 
participants randomly assigned to the three conditions of the Learned Helplessness Task. We next conducted a separate 3 (Condition) $\times 5$ (Time-Bin) mixed-design analysis of variance (ANOVA) on PFTA and frontal EEG alpha asymmetry. Condition, a between-subjects factor, involved participants in the Uncontrollable, Controllable, or No-Noise groups. Time-Bin, a within-subjects factor, involved dividing the 50 trials of the Learned Helplessness Task into five time-bins of ten trials each $($ Bin $1=$ trials $1-10$, Bin $2=$ trials $11-20$, Bin 3 $=$ trials $21-30$, Bin $4=$ trials $32-40$, Bin $5=$ trials $41-50$ ). This Time-Bin structure allowed us to track the slope of PFTA over time (i.e., temporal dynamics) as participants in the Uncontrollable Condition were exposed to progressively more uncontrollable aversive stimuli. In other words, the withinsubjects factor of Time-Bin allowed us to examine the time course or temporal dynamics of perceived uncontrollability on EEG activity, and approach motivation more generally. We decided on ten trials per time bin because previous research indicates that ten trials generate a reliable index of EEG activity (David \& Friston, 2003; Harmon-Jones et al., 2008). Thus, five time-bins of ten trials each maximized our ability to examine the temporal dynamics of perceived uncontrollability and learned helplessness induction while still having a reliable EEG index for each time-bin. Effect sizes are reported as the proportion of variance accounted for $\left(\eta_{p}{ }^{2}\right)$.

When we observed a significant main effect of either Condition or Time-Bin, or a Condition by Time-Bin interaction, we re-ran the planned analysis adjusting for resting levels of PFTA to determine whether the effect was present above and beyond pre-existing individual differences in EEG activity.

Examining the independent contribution of anterior versus posterior theta activity The PFTA difference score is limited by its inability to speak to whether the difference score is driven primarily by $\mathrm{PZ}$ (posterior) theta activity, FZ (anterior) theta activity, or both. For example, reduced PFTA could be driven by reduced theta activity at PZ or increased theta activity at FZ. To address this limitation, event-related desynchronization (ERD) analyses were conducted in the case of a significant main effect or interaction on PFTA to examine the independent contribution of PZ versus FZ theta activity. ERD represents a power decrease at a given frequency relative to power at that frequency during a reference period (Pfurtscheller, 2001). In the present study, the ratio of theta power during the Learned Helplessness Task relative to theta power at rest was calculated as an ERD index. This ratio was calculated by dividing the natural log of activity during the controllability task by resting state activity [ $\ln$ (theta power during task/theta power during resting state)] as in prior research (Pfurtscheller, 2001). Higher values on this index indicate an increase in power, whereas lower values indicate a decrease. ERD allows researchers to infer the potential sites that may be driving an effect. In the present study, we wanted to examine whether effects may be driven in changes in theta activity at posterior $(\mathrm{PZ})$ versus anterior $(\mathrm{FZ})$ sites.

Examining the independent contribution of left versus right frontal alpha activity The limitations of the PFTA difference score also apply to the frontal EEG alpha asymmetry index, which involves a difference score of right minus left alpha power for homologous electrodes. In the case of a significant main effect or interaction with frontal EEG alpha asymmetry, ERD analyses were conducted to examine the independent effect of left versus right alpha power. The ERD index for alpha power analyses was calculated by dividing the natural $\log$ of alpha power during the controllability task by resting state alpha power [ln(alpha power during task/ alpha power during resting state)].

\section{Results}

\section{Perceived uncontrollability and approach motivation manipulation check}

Consistent with expectation, participants in the Uncontrollable Condition of the Learned Helplessness Task reported significantly lower perceived control $(M=1.13, S D=0.46)$ over the duration of the noise bursts than participants in the Controllable Condition $(M=3.71, S D=1.31), t(42)=-8.90, p=0.001$. Also consistent with expectation, participants in the Uncontrollable Condition reported significantly lower motivation $(M=3.17, S D=1.40)$ to find the response pattern to turn off the noise bursts than participants in the Controllable Condition $(M=4.38, S D=.81), t(42)=-3.46, p=0.01$.

\section{Effect of learned helplessness on posterior versus anterior theta activity (PFTA)}

As expected, participants in the Uncontrollable, Controllable, and No-Noise Conditions did not differ on PFTA at rest, $F(2,71)=.47, p=0.63$, indicating that participants in these three conditions had equivalent profiles of PFTA at the outset of the study.

There was a main effect of Time-Bin on PFTA, $F(4,71)=$ 2.61, $p=.04, \eta_{p}{ }^{2}=.04$ (Fig. 1). Follow-up contrasts revealed that PFTA levels during both Time-Bin $3(M=.20, S D=.43)$ and Time-Bin $4(M=.22, S D=.37)$ were significantly lower than PFTA levels during Time-Bin $1(M=.28, S D=.33)$, all $p$ $<.05$, and that PFTA during Time-Bin $5(M=.23, S D=.40)$ was marginally lower than Time-Bin $1, p<.09$. There was no significant difference in PFTA levels between Time-Bins 1 and $2(M=.27, S D=.36)$, nor between Time-Bins 2 through 5 , all $p>.20$. This main effect of Time-Bin on PFTA was maintained after controlling for resting PFTA, $F(2,70)=$ 
PZ-FZ Theta Means Over Time Course of Controllability Task

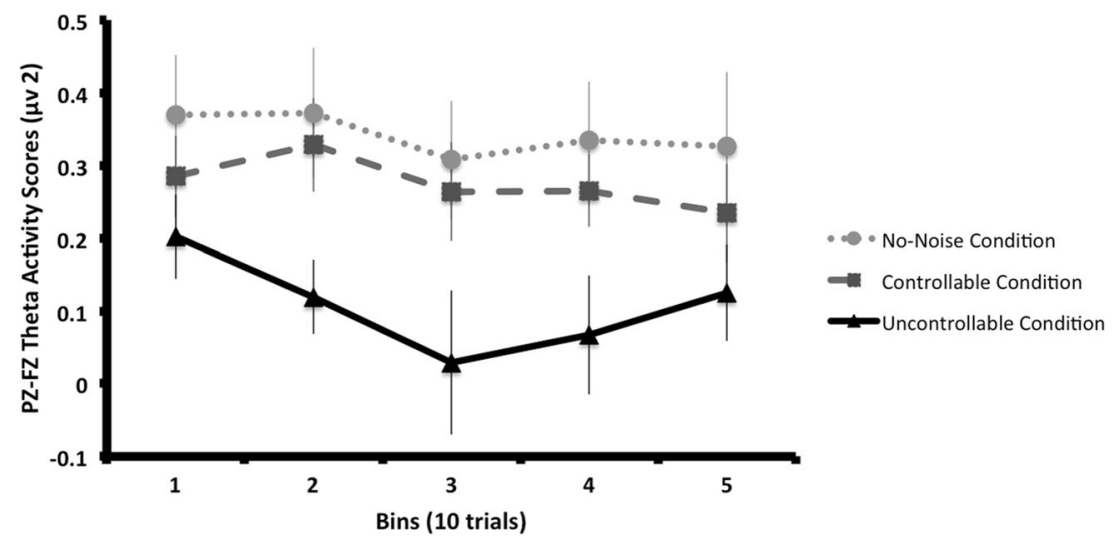

Fig. 1 Posterior versus frontal theta activity (PFTA) during the Learned Helplessness Task for Uncontrollable, Controllable, and No-Noise Conditions. Error bars denote 1 standard error

4.76, $p=0.01, \eta_{p}^{2}=0.12$. Furthermore, follow-up contrasts revealed that after controlling for resting PFTA, Time Bin 3 ( $M$ $=.20, S E=.04)$ and Time Bin $4(M=.22, S E=.03)$ still had significantly lower levels of PFTA than Time Bin $1(M=.28$, $S E=.02)$. Time $\operatorname{Bin} 3(M=.20, S E=.04)$ also had significantly lower levels of PFTA than Time Bin $2(M=.27, S E=.03), p<$ .05 after controlling for resting PFTA. There was no significant difference between Bin 2 and Bins 1, 4, and 5, $p>.102$, after controlling for resting PFTA, and there was also no significant difference between Bin 3 and Bins 4 and 5, $p>$.34. There were no significant differences between Bin 5 and any of the other Bins, $p>.09$, after controlling for resting PFTA. There was a potential trend level difference between $\operatorname{Bin} 5(M=.23, S E=$ $.04)$ and Bin $1, p=.09$.

In line with prediction, there was also a main effect of Condition on PFTA, $F(2,71)=3.29, p=0.04, \eta_{p}{ }^{2}=.09$ (Fig. 1). Follow-up contrasts revealed that participants in the Uncontrollable Condition $(M=.10, S D=.35)$ had significantly lower PFTA levels than participants in the No-Noise Condition $(M=.34, S D=.41), p=.02$, and marginally lower PFTA levels than participants in the Controllable Condition $(M=.27, S D=.29), p=.07$. There was no difference in PFTA levels between participants in the Controllable and No-Noise Conditions, $p=$ .5. This main effect of Condition was maintained after controlling for resting PFTA, $F(2,70)=4.76, p=0.01$, $\eta_{p}{ }^{2}=0.12$. Furthermore, follow-up contrasts revealed that after controlling for resting PFTA, participants in the Uncontrollable Condition $(M=.13, S E=.04)$ still had significantly lower PFTA levels than participants in the Controllable Condition $(M=.29, S E=.04), p=.014$ and No-Noise Condition $(M=.30, S E=.04), p=.008$. Finally, among participants in the Uncontrollable Condition, lower PFTA levels during the Learned Helplessness Task was associated with decreased selfreported motivation to identify the response pattern that turned off the noise burst, after controlling for resting
PFTA, $\beta=.43, t(20)=2.14, p=.04$. There was no significant difference in PFTA levels in individuals in the Controllable and No-Noise Conditions, $\mathrm{p}=.78 .^{1}$

Examining the independent contribution of anterior versus posterior theta activity Event-related desynchronization (ERD) analyses were utilized to investigate the independent contribution of PZ and FZ theta activity to the PFTA difference score for the aforementioned results (see Fig. 2). With respect to PZ, there was no significant main effect of Time-Bin, $F(4,71)=$ $.68, p=.61, \eta_{p}{ }^{2}=.01$. There was, however, a non-significant trend of Condition, $F(2,71)=2.35, p=.10, \eta_{p}{ }^{2}=.06$, and a significant Condition by Time-Bin interaction, $F(8,71)=2.20$, $p=.03, \eta_{p}{ }^{2}=.03$. PZ theta activity differed significantly by Condition at Time-Bin 2, $F(2,71)=3.86, p=.03, \eta_{p}{ }^{2}=.10$, and trended towards a significant difference at Bins $3, F(2,71)=$ 2.51, $p=.09, \eta_{p}{ }^{2}=.07$ and $4, F(2,71)=2.49, p=.09, \eta_{p}{ }^{2}=$

\footnotetext{
${ }^{1}$ To assess the specificity of the effect at the theta band, we also examined the difference between alpha $(8-12 \mathrm{~Hz})$, beta $(13-30 \mathrm{~Hz})$, and delta $(1-3 \mathrm{~Hz})$ activity at $\mathrm{PZ}$ and FZ. The difference score for these frequency bands was computed in the same manner as the theta band $([\ln (\mathrm{Pz})-\ln (\mathrm{Fz})]$. There was no main effect of Condition on the PZ-FZ difference score at the alpha band, $\mathrm{F}(2,71)=.85, p=.42$ or at the beta band, $\mathrm{F}(2,71)=.63, p=.54$. There was, however, a non-significant trend level effect of Condition on the PZ-FZ difference score at the delta band, $\mathrm{F}(2,71)=2.76, p=.070, \eta_{p}{ }^{2}=.072$. Collectively, these findings suggest that the effect of the Learned Helplessness Task on the PZ-FZ difference score is most prominent at the delta-theta bands. Particularly, in two EEG studies with relatively large sample sizes, $(\mathrm{N}=167$; Wacker, Chavanon, \& Stemmler, 2010) and $(\mathrm{N}=$ 78; Chavanon, Wacker, \& Stemmler, 2011), both PZ-FZ delta and PZ-FZ theta indices at rest significantly predicted extraversion in the same direction as reported in the present study. However, PZ-FZ delta had a smaller effect size than PZFZ theta in these studies, which may explain the trend in our delta-band finding. Moreover, in addition to extraversion, both PZ-FZ theta and PZ-FZ delta indices also have been related to a polymorphism of COMT, the enzyme catechol-Omethyltransferase that is involved in breaking down dopamine (Wacker \& Gatt, 2010). More recently, time-frequency studies also report enhanced parietal delta activity to reward-related cues and feedback (Cavanagh, 2015; Foti, Weinberg, Bernat, \& Proudfit, 2015; Pornpattananangkul \& Nusslock, 2016) that may be related to parietal delta indices found in our current study. As such, these results suggest that further exploration of PZ-FZ activity in both the theta and delta frequency bands is warranted.
} 


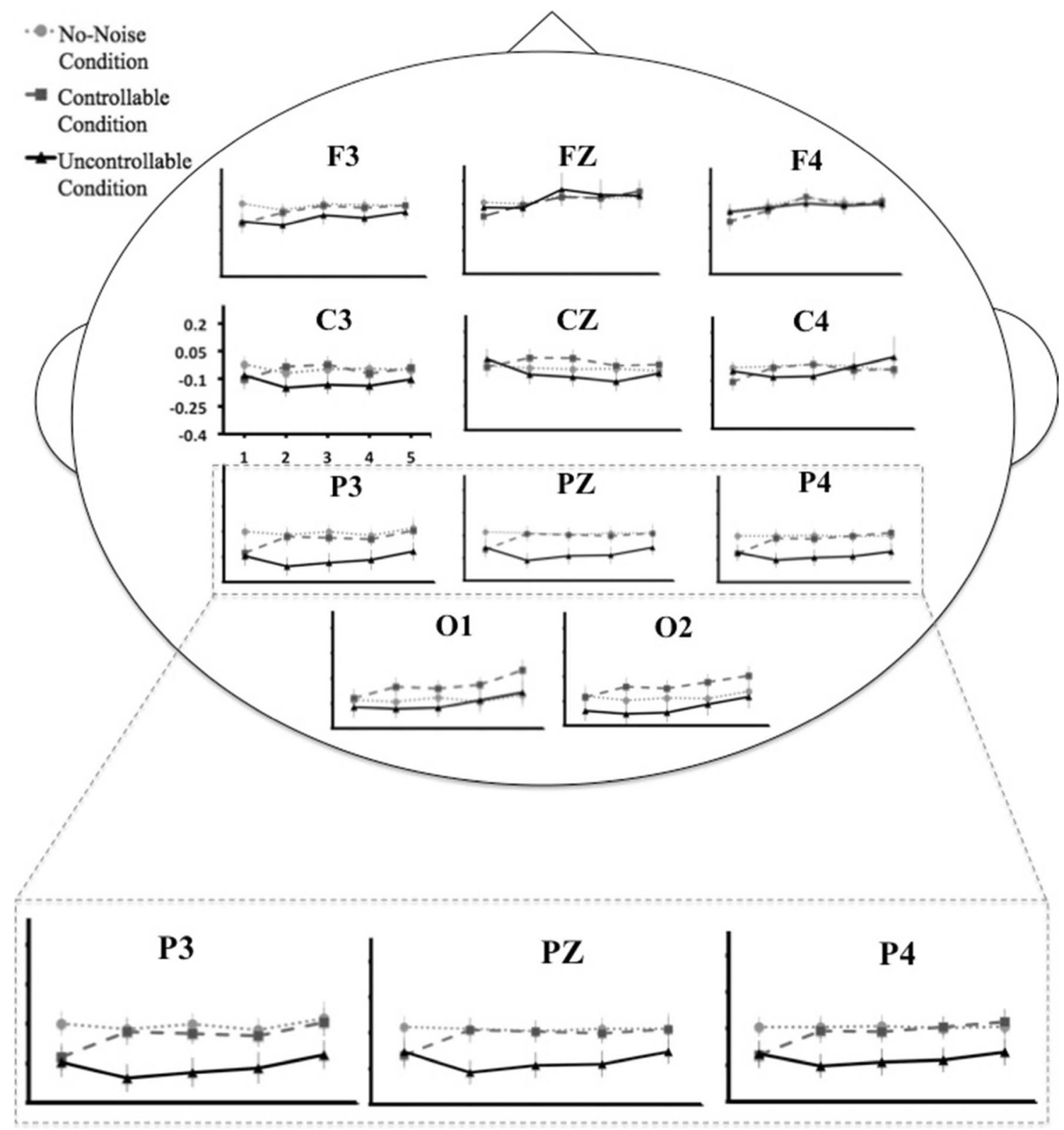

Fig. 2 Event-related desynchronization (ERD) analyses were utilized to investigate the independent contribution of $\mathrm{PZ}$ and FZ theta activity to the posterior versus frontal theta activity (PFTA) difference score. ERD analyses at selected electrodes surrounding $\mathrm{PZ}$ and $\mathrm{FZ}$ are displayed in the

.07. Follow-up contrasts indicated that the Uncontrollable Condition had significantly lower PZ theta values than the No-Noise Condition across Bins 2, $p<.02,3, p<.05$, and 4 , $p<.04$, and the Controllable Condition at Bin 2, $p<.03$. Trends existed for lower PZ theta values in the Uncontrollable than Controllable Condition at $\operatorname{Bin} 3, p<.07$, and 4, $p<.10$, whereas there were no differences between the Controllable and NoNoise Conditions across Bins 2, 3, 4, and 5 (all $p>.57$ ).

With respect to FZ, there was no main effect of Condition, $F(2,71)=.44, p=.65, \eta_{p}{ }^{2}=.01$ and no Condition by Time-Bin interaction, $F(8,71)=.57, p=.80, \eta_{p}{ }^{2}=.02$. There was a significant main effect of Time-Bin for $\mathrm{FZ}, F(4,71)=4.08, p$ figure above. Theta activity is graphed at each of the five time bins (ten trials per bin) during the Learned Helplessness Task. Error bars denote 1 standard error

$=.003, \eta_{p}{ }^{2}=.05$. Follow-up analyses indicated that FZ theta activity was greater at Bin 1 compared to Bin $5(p=.01)$. Collectively, ERD analyses suggest that the main effect of Condition on PFTA observed in the present study was primarily driven by posterior (PZ) rather than frontal (FZ) theta activity.

\section{Effect of learned helplessness on frontal EEG alpha asymmetry}

As expected, participants in the Uncontrollable, Controllable, and No-Noise Conditions did not differ on frontal EEG alpha 
asymmetry at rest, $F(2,71)=1.6, p=.21$, indicating that participants in these three conditions had equivalent profiles of frontal EEG alpha asymmetry at the outset of the study.

Contrary to prediction, there was no main effect of Condition, $F(2,71)=1.31, p=.28, \eta_{p}{ }^{2}=.035$, no main effect of Time-Bin, $F(4,71)=1.11, p=.35, \eta_{p}^{2}=.02$, and no Condition by Time-Bin interaction, $F(8,71)=1.15, p=.33$, $\eta_{p}{ }^{2}=.031$, for frontal EEG alpha asymmetry. Given these non-significant findings with the asymmetry indices, we did not conduct follow-up ERD analyses for frontal alpha power. ${ }^{2}$

\section{Discussion}

Growing evidence indicates that midline posterior versus frontal EEG theta activity (PFTA) reflects a novel neurophysiological index of approach motivation (Wacker et al., 2006, 2010; Walden et al., 2015). Research has yet to examine, however, PFTA during a laboratory task designed to reduce or attenuate approach motivation. Accordingly, the present study examined the effect of perceived uncontrollability on midline posterior versus frontal EEG theta activity. Considerable animal and human research supports the hypothesis put forth by the Learned Helplessness Theory that exposure to uncontrollable aversive stimuli is associated with reduced approach motivation and decreased goal-directed behaviors (Abramson et al., 2002; Alloy et al., 1984; Grahn et al., 2000; Hiroto \& Seligman, 1975; Miller \& Seligman, 1975). In line with prediction, participants exposed to uncontrollable aversive noise bursts displayed a significant decrease in PFTA, reflecting decreased approach motivation, relative to both individuals exposed to controllable noise bursts and participants in the No-Noise Condition. This study is the first to report decreased PFTA during a laboratory task designed to attenuate approach motivation and goal-directed behavior. Contrary to prediction, results were specific to PFTA, as there was no relationship between perceived uncontrollability and frontal EEG alpha asymmetry.

Previous research has focused on resting PFTA as an index of approach tendencies (Wacker et al., 2006, 2010). In these studies, elevated PFTA at rest was presumed to indicate a dispositional tendency toward approach-related behavior without regard for situational demands. Dispositional models of personality contend that general tendencies hold across a variety of situations (Costa Jr. \& McCrae, 2009). However, situational models propose that an individual's behavior will vary in response to the demands of the situation (Mischel \& Shoda, 2010), and that emotionally evocative tasks may yield

\footnotetext{
${ }^{2}$ Contrary to previous literature, there was no significant relationship between PFTA or frontal EEG alpha asymmetry either at rest or during the Learned Helplessness Task and self-reported behavioral approach system (BAS) or behavioral inhibition system (BIS) sensitivity, as measured by Carver \& White's (1994) BIS/BAS scale, $p>.17$.
}

a more pure measure of neurophysiological individual differences than resting state data (Coan et al., 2006). Accordingly, researchers have begun to examine PFTA during laboratory tasks designed to modulate approach-related tendencies. In the first such study, Walden and colleagues (2015) reported elevated PFTA during an autobiographical memory task designed to increase approach motivation. The present study extends this work by reporting, for the first time, that PFTA decreases during a learned helplessness induction task designed to reduce or attenuate approach motivation and goaldirected behavior (i.e., perceived uncontrollability over an aversive stimulus). Collectively, this work suggests that PFTA is a promising neurophysiological index of both elevated and reduced approach motivational tendencies.

We argue that decreased PFTA among participants in the Uncontrollable Condition reflected a laboratory-induced state of learned helplessness in which these participants came to expect that their responses and the termination of the aversive noise were independent of each other. Previous research using the identical Learned Helplessness Task employed in the present study has demonstrated that this perception of uncontrollability results in reduced response initiation and behavioral disengagement as participants in the Uncontrollable Condition are no longer searching for solutions to turn off the noise and no longer attempting to exert control over their circumstances (Alloy et al., 1984; Hiroto \& Seligman, 1975; Miller \& Seligman, 1975). This behavioral disengagement, from this perspective, is associated with helplessness and a general reduction in approach motivation (see Abramson et al., 1978, 1989, 2002 for reviews). The present study is the first to report that the decreased approach motivation documented to occur during the Learned Helplessness Task (Alloy et al., 1984; Hiroto \& Seligman, 1975; Miller \& Seligman, 1975) is reflected in reduced PFTA. ${ }^{3}$ The fact that PFTA was modulated by a laboratory-based learned helplessness task suggests that it may be a useful marker for understanding the pathophysiology of depression and, in particular, depressive symptoms characterized by reduced approach motivation and behavioral disengagement, such as anhedonia, decreased energy, and psychomotor retardation. Future research is needed to test this hypothesis.

A strength of PFTA as a neurophysiological index is that it has been linked to dopamine-related neural activity in the rACC (Wacker et al., 2006; Wacker, Chavanon, et al., 2010), which animal and human research indicates is central to reward processing and approach-related motivation (Berridge, 2007; Berridge et al., 2009; Haber \& Knutson, 2010; Kringelbach \& Berridge, 2010; Schultz, 2002). This link

\footnotetext{
${ }^{3}$ An alternative explanation for reduced PFTA in the Uncontrollable Condition is that participants in this condition found the Learned Helplessness Task to be less interesting or engaging, rather than it inducing a state of learned helplessness, per se. Given that we did not directly assess task engagement, future research is needed to examine this competing hypothesis.
} 
validates PFTA as indexing critical reward-related neural processes and provides PFTA with a neurobiological foundation that is atypical of neurophysiological measures. Furthermore, elevated PFTA at rest has also been associated with elevated self-reported behavioral approach system sensitivity (BAS) (Wacker et al., 2010), whereas reduced resting delta/theta current in the rACC is associated with anhedonia, which involves reduced approach motivation and decreased interest or pleasure in typically rewarding activities (Wacker et al., 2009). This link between PFTA and both reward-related brain function and approach-related tendencies suggests that PFTA is likely an index of approach motivational tendencies as opposed to avoidance or withdrawal tendencies. Additionally, there is considerable evidence summarized in the present paper that perceived uncontrollability over aversive stimuli - as reflected in Learned Helplessness Theory - decreases approach motivation, reduces goal-directed behaviors, and attenuates reward-related neural activation in the ventral striatum (Abramson et al., 1978, 2002; Hiroto \& Seligman, 1975; Salomons et al., 2015). In line with this perspective, participants in the Uncontrollable Condition in the present study reported significantly lower motivation to identify the response pattern that turned off the noise bursts than participants in the Controllable Condition. This suggests that the Uncontrollable Condition of the Learned Helplessness Task was effective in reducing approach motivation. Furthermore, among participants in the Uncontrollable Condition, lower self-reported motivation to identify the response pattern was associated with lower PFTA levels, after controlling for PFTA levels at rest. Accordingly, we argue that the profile of reduced PFTA among participants in the Uncontrollable Condition reflects reduced approach motivation rather than elevated aversion or withdrawal motivation. However, completely dissociating reduced approach motivation from elevated aversion is challenging, and we need future research assessing both approach motivation and aversion to fully address whether decreased PFTA involves reduced approach motivation, elevated aversion, or some combination of the two.

The reduction in PFTA among participants in the Uncontrollable Condition during the Learned Helplessness Task indicated a change in the relative relationship (i.e., difference score) between PZ and FZ theta activity. The PFTA difference score, however, is limited by its inability to speak to whether this effect is primarily driven by $\mathrm{PZ}$ (posterior) theta activity, FZ (anterior) theta activity, or both. To address this issue, we conducted event-related desynchronization (ERD) analyses to examine the independent contribution of $\mathrm{PZ}$ versus FZ theta activity for all significant main effects and interactions. ERD analyses indicated a main effect of Condition and a Condition by Time-Bin interaction at PZ but not FZ. Follow-up analyses indicated that these effects were driven by reduced $\mathrm{PZ}$ theta values among participants in the Uncontrollable Condition over time, suggesting that the condition difference in PFTA observed in the present study was primarily driven by posterior rather than frontal theta activity. $^{4}$ This finding is consistent with a previous study reporting that agentic extraversion, characterized by elevated approach motivation, is associated with increased theta activity at posterior sites (Knyazev, Bocharov, \& Pylkova, 2012). As argued by Luck and colleagues (Luck, 2014), however, inferring the exact anatomical location of an EEG signal is difficult due to the inverse problem. That is, a large number of different sets of dipoles in the brain can lead to the same distribution of scalp EEG. Because we measured the distribution of EEG on the scalp, it is hard for us to determine which sets of the dipoles in the brain lead to the scalp EEG in our data. Estimating the source of PZ or FZ theta activity requires confirmation from other techniques, such as placing EEG sensors inside the brain as in electrocorticography (ECoG) and simultaneous EEG-fMRI recording. Nonetheless, we argue that our ERD analysis provides a stronger understanding of the topographical distribution of theta activity over the scalp. ERD analyses allow us to infer, for instance, that based on the distribution of theta power and the direction of the effect, PFTA profiles assessed in the present study appear to index separable psychological phenomena from frontal-midline theta. That is, frontal-midline theta is typically distributed at frontal sites and is enhanced by negative feedback (Cavanagh \& Shackman, 2015; Cohen, Wilmes, \& van de Vijver, 2011; Luft, 2014; Pornpattananangkul \& Nusslock, 2016). As PFTA in the present study was driven by the posterior site and reduced by negative feedback, it appears to be a distinct neurophysiological index. That is, PFTA changes in the present study were driven by activity at the posterior sites in response to negative feedback, whereas frontal-midline theta is typically distributed at frontal sites and is enhanced, not reduced, by negative feedback.

It is also important to note that most of the PFTA studies conducted thus far (mainly done by Wacker and colleagues, 2006; 2010) focus on PFTA at rest. During studies of resting neurophysiological activity there typically is not a separate baseline to compute an ERD, and thus researchers have to rely on the PFTA difference score. The present study is among the first to show that PFTA may be driven primarily by posterior rather than frontal theta activity, thereby deepening our understanding of neurophysiological mechanisms underlying PFTA. Future research is needed to examine whether the topographical contribution of posterior versus anterior theta activity observed in the present study is specific to laboratory tasks designed to reduce approach motivation, or whether this

\footnotetext{
${ }^{4}$ Reduced PZ theta activity in follow-up ERD analyses among participants in the Uncontrollable versus Controllable Condition was a non-significant trend at time-bins 2 and 3 ( $p s=.09$ ). These findings should be interpreted cautiously and future research with greater statistical power is required to confirm these effects.
} 
distribution also occurs when approach motivation is enhanced.

We included Time Bin as a factor so we could model PFTA over the time course of learned helplessness onset via exposure to progressively more uncontrollable aversive stimuli. In line with previous research (Mikulincer, 1988), it took time for participants in the Uncontrollable Condition to display decreased approach motivation, as reflected in reduced PFTA. Specifically, participants in the Uncontrollable Condition did not display reduced PFTA until the second time bin (trials 11$20)$ and this reduction continued through Time-Bin 3. Contrary to prediction, PFTA levels began to normalize among participants in the Uncontrollable Condition in TimeBin 5 (although PFTA levels during Time-Bin 5 were not significantly different from PFTA levels during either TimeBin 3 or Time-Bin 4 among participants in the Uncontrollable Condition, all $p>.15$ ). It is unclear whether this normalization would have continued during subsequent time-bins (perhaps reflecting habituation) or whether it represents less meaningful variation. Future research with more prolonged exposure to uncontrollable aversive stimuli is needed to better understand the temporal dynamics of laboratory induced learned helplessness on PFTA.

Contrary to expectation, perceived uncontrollability did not modulate frontal EEG alpha asymmetry, another frequently used neurophysiological index of approach motivation. Research on frontal alpha asymmetry has typically examined this index at rest (see Allen \& Reznik, 2015; Coan \& Allen, 2004; Nusslock, Walden, \& Harmon-Jones, 2015 for reviews), during laboratory paradigms designed to elevate approach motivation [(e.g., reward/monetary cues (Miller \& Tomarken, 2001; Sobotka, Davidson, \& Senulis, 1992); anger provocation (Harmon-Jones et al., 2010 for review)], or during paradigms designed to elevate avoidance or aversive emotional states [e.g., viewing negative pictures (Jackson et al., 2003; Petrantonakis \& Hadjileontiadis, 2011); anticipating public speaking (Davidson, Marshall, Tomarken, \& Henriques, 2000)]. To the best of our knowledge, no studies have examined frontal alpha asymmetry during laboratory tasks specifically designed to reduce approach motivation or induce a state of learned helplessness in a manner such as the present study. Although it is difficult to interpret a null effect, one possibility is that, whereas PFTA is sensitive to both elevated and attenuated approach motivational tendencies, frontal asymmetry is more impacted by laboratory tasks than enhance approach motivation. Future research is needed to test this hypothesis.

\section{Conclusion}

The present study, for the first time, reports that a learned helplessness task (i.e., perceived uncontrollability over an aversive stimulus) is associated with a corresponding reduction in PFTA. Results were specific to PFTA, as there was no relationship between frontal EEG alpha asymmetry and perceived uncontrollability. When combined with existing research, the results of the present study suggest that PFTA is sensitive to both attenuated and elevated approach motivational states (Walden et al., 2015), as well as dispositional approach-motivational tendencies as indexed by resting PFTA (Wacker, Chavanon, et al., 2010). Follow-up ERD analyses indicated that PFTA findings in the present study were driven by reduced posterior (PZ) theta values among participants in the Uncontrollable Condition. This finding suggests that posterior theta activity may be particularly important for understanding the neurophysiology of decreased approach motivation and reduced goal-directed behavior. A strength of PFTA as a neurophysiological index of approach motivation is that it has been directly linked to dopamine signaling in the rACC, which animal and human research indicates is central to reward processing and approach-related motivation. This link validates PFTA as indexing critical reward-related neural processes, and provides PFTA with a neurobiological foundation that is atypical of other neurophysiological measures.

\section{References}

Abramson, L. Y., Alloy, L. B., Hankin, B. L., Haeffel, G. J., MacCoon, D. G., Gibb, B. E., \& Gotlib, I. H. (2002). Cognitive vulnerabilitystress models of depression in a self-regulatory and psychobiological context. In I.H. Gotlib and C.L. Hammen (Eds.): Handbook of depression (pp. 268-294). http://doi.org/10.1023/A

Abramson, L. Y., Metalsky, G. I., \& Alloy, L. B. (1989). Hopelessness depression: A theory-based subtype of depression. Psychological Review, 2, 358-372. doi:10.1037/0033-295X.96.2.358

Abramson, L. Y., Seligman, M. E. P., \& Teasdale, J. D. (1978). Learned helplessness in humans: Critique and reformulation. Journal of Abnormal Psychology, 87(1), 49-74. Retrieved from http://www. ncbi.nlm.nih.gov/pubmed/649856

Allen, J. J. B., Coan, J. A., \& Nazarian, M. (2004). Issues and assumptions on the road from raw signals to metrics of frontal EEG asymmetry in emotion. Biological Psychology, 67, 183-218. doi:10. 1016/j.biopsycho.2004.03.007

Allen, J. J. B., \& Reznik, S. J. (2015). Frontal EEG asymmetry as a promising marker of depression vulnerability: Summary and methodological considerations. Current Opinion in Psychology, 4, 9397. doi:10.1016/j.copsyc.2014.12.017

Allman, J. M., Hakeem, A., Erwin, J. M., Nimchinsky, E., \& Hof, P. (2001). The anterior cingulate cortex: The evolution of an interface between emotion and cognition. Annals of the New York Academy of Sciences, 935, 107-17. Retrieved from http://www.ncbi.nlm.nih. gov/pubmed/11411161

Alloy, L. B., Peterson, C., Abramson, L. Y., \& Seligman, M. E. (1984). Attributional style and the generality of learned helplessness. Journal of Personality and Social Psychology, 46(3), 681-7. Retrieved from http://www.ncbi.nlm.nih.gov/pubmed/6707869

Baker, T. E., \& Holroyd, C. B. (2011). Dissociated roles of the anterior cingulate cortex in reward and conflict processing as revealed by the 
feedback error-related negativity and N200. Biological Psychology, 87(1), 25-34. doi:10.1016/j.biopsycho.2011.01.010

Berridge, K. C. (2007). The debate over dopamine's role in reward: The case for incentive salience. Psychopharmacology, 191(3), 391-431. doi:10.1007/s00213-006-0578-x

Berridge, K. C., Robinson, T. E., \& Aldridge, J. W. (2009). Dissecting components of reward: "liking", "wanting", and learning. Current Opinion in Pharmacology, 9(1), 65-73. doi:10.1016/j.coph.2008. 12.014

Bland, S. T., Hargrave, D., Pepin, J. L., Amat, J., Watkins, L. R., \& Maier, S. F. (2003). Stressor controllability modulates stress-induced dopamine and serotonin efflux and morphine-induced serotonin efflux in the medial prefrontal cortex. Neuropsychopharmacology, 28(9), 1589-1596. doi:10.1038/sj.npp.1300206

Blasi, G., Mattay, V. S., Bertolino, A., Elvevåg, B., Callicott, J. H., Das, S., ... Weinberger, D. R. (2005). Effect of catechol-Omethyltransferase val158met genotype on attentional control. The Journal of Neuroscience, 25(20), 5038-5045. http://doi.org/10. 1523/JNEUROSCI.0476-05.2005

Carver, C. S., \& White, T. L. (1994). Behavioral inhibition, behavioral activation, and affective responses to impending reward and punishment: The BIS/BAS Scales. Journal of Personality and Social Psychology, 67(2), 319-333. doi:10.1037//0022-3514.67.2.319

Cavanagh, J. F. (2015). Cortical delta activity reflects reward prediction error and related behavioral adjustments, but at different times. NeuroImage, 110, 205-216. doi:10.1016/j.neuroimage.2015.02.007

Cavanagh, J. F., \& Shackman, A. J. (2015). Frontal midline theta reflects anxiety and cognitive control: meta-analytic evidence. Journal of Physiology, Paris, 109(1), 3-15. doi:10.1016/j.jphysparis.2014.04.003

Chapman, L. J., \& Chapman, J. P. (1987). The measurement of handedness. Brain and Cognition, 6(2), 175-183. doi:10.1080/08856559. 1933.10533126

Chavanon, M., Wacker, J., \& Stemmler, G. (2011). Rostral anterior cingulate activity generates posterior versus anterior theta activity linked to agentic extraversion. Cognitive, Affective, \& Behavioral Neuroscience, 11(2), 172-185. doi:10.3758/s13415-010-0019-5

Chavanon, M., Wacker, J., \& Stemmler, G. (2013). Paradoxical dopaminergic drug effects in extraversion: Dose- and time-dependent effects of sulpiride on EEG theta activity. Frontiers in Human Neuroscience, 7, 1-15. doi:10.3389/fnhum.2013.00117

Coan, J. A., \& Allen, J. J. B. (2004). Frontal EEG asymmetry as a moderator and mediator of emotion. Biological Psychology, 67(1-2), 749. doi:10.1016/j.biopsycho.2004.03.002

Coan, J. A., Allen, J. J. B., \& McKnight, P. E. (2006). A capability model of individual differences in frontal EEG asymmetry. Biological Psychology, 72(2), 198-207. doi:10.1016/j.biopsycho.2005.10.003

Cohen, M. X., Wilmes, K., \& van de Vijver, I. (2011). Cortical electrophysiological network dynamics of feedback learning. Trends in Cognitive Sciences, 15(12), 558-566. doi:10.1016/j.tics.2011.10.004

Costa Jr., P., \& McCrae, R. (2009). The Five-Factor Model and the NEO Inventories. Oxford Handbook of Personality Assessment., 299-322.

David, O., \& Friston, K. J. (2003). A neural mass model for MEG/EEG: Coupling and neuronal dynamics. NeuroImage, 20(3), 1743-1755. doi:10.1016/j.neuroimage.2003.07.015

Davidson, R. J. (1994). Asymmetric brain function, affective style, and psychopathology: The role of early experience and plasticity. http:// doi.org/10.1017/S0954579400004764

Davidson, R. J. (1998). Anterior electrophysiological asymmetries, emotion, and depression: Conceptual and methodological conundrums. Psychophysiology, (September 1998), 607-614. Retrieved from http://journals.cambridge.org/abstract_S0048577298000134

Davidson, R. J., Marshall, J. R., Tomarken, A. J., \& Henriques, J. B. (2000). While a phobic waits: Regional brain electrical and autonomic activity in social phobics during anticipation of public speaking. Biological Psychiatry, 47(2), 85-95. doi:10.1016/S00063223(99)00222-X
Foti, D., Weinberg, A., Bernat, E. M., \& Proudfit, G. H. (2015). Anterior cingulate activity to monetary loss and basal ganglia activity to monetary gain uniquely contribute to the feedback negativity. Clinical Neurophysiology, 126(7), 1338-1347. doi:10.1016/j. clinph.2014.08.025

Gottesman, I. I., \& Gould, T. D. (2003). The endophenotype concept in psychiatry: Etymology and strategic intentions. American Journal of Psychiatry, 160(4), 636-645. doi:10.1176/appi.ajp.160.4.636

Grahn, R. E., Watkins, L. R., \& Maier, S. F. (2000). Impaired escape performance and enhanced conditioned fear in rats following exposure to an uncontrollable stressor are mediated by glutamate and nitric oxide in the dorsal raphe nucleus. Behavioural Brain Research, 112(1-2), 33-41. doi:10.1016/S0166-4328(00)00161-3

Haber, S. N., \& Knutson, B. (2010). The reward circuit: linking primate anatomy and human imaging. Neuropsychopharmacology, 35(1), 4-26. doi:10.1038/npp.2009.129

Harmon-Jones, E. (2004). On the relationship of frontal brain activity and anger: Examining the role of attitude toward anger. Cognition \& Emotion, 18(3), 337-361. doi:10.1080/02699930341000059

Harmon-Jones, E., Abramson, L. Y., Nusslock, R., Sigelman, J. D., Urosevic, S., Turonie, L. D., ... Fearn, M. (2008). Effect of Bipolar Disorder on Left Frontal Cortical Responses to Goals Differing in Valence and Task Difficulty. Biological Psychiatry, 63(7), 693-698. http://doi.org/10.1016/j.biopsych.2007.08.004

Harmon-Jones, E., Gable, P. A., \& Peterson, C. K. (2010). The role of asymmetric frontal cortical activity in emotion-related phenomena: a review and update. Biological Psychology, 84(3), 451-62. doi:10. 1016/j.biopsycho.2009.08.010

Hayden, B. Y., Pearson, J. M., \& Platt, M. L. (2009). Fictive reward signals in the anterior cingulate cortex. Science, 324(5929), 94850. doi: $10.1126 /$ science. 1168488

Hiroto, D. S., \& Seligman, M. E. (1975). Generality of learned helplessness in man. Journal of Personality and Social Psychology, 31(2), 311-327. doi:10.1037/h0076270

Huang, J. J., Yen, C. T., Liu, T. L., Tsao, H. W., Hsu, J. W., \& Tsai, M. L. (2013). Effects of dopamine D2 agonist quinpirole on neuronal activity of anterior cingulate cortex and striatum in rats. Psychopharmacology, 227(3), 459-466. doi:10.1007/s00213-0132965-4

Jackson, D. C., Mueller, C. J., Dolski, I., Dalton, K. M., Nitschke, J. B., Urry, H. L., ... Davidson, R. J. (2003). Now you feel it, now you don't: Front brain electrical assymetry and individual differences in emotion regulation. Psychological Science, 14(6), 612-617. doi:10. 1046/j.0956-7976.2003.psci 1473.x

Knyazev, G. G., Bocharov, A. V., \& Pylkova, L. V. (2012). Extraversion and fronto-posterior EEG spectral power gradient: An independent component analysis. Biological Psychology, 89(2), 515-524. doi: 10.1016/j.biopsycho.2011.12.023

Koehler, S., Wacker, J., Odorfer, T., Reif, A., Gallinat, J., Fallgatter, A. J., \& Herrmann, M. J. (2011). Resting posterior minus frontal EEG slow wave oscillations is associated with extraversion and DRD2 genotype. Biological Psychology, 87, 407-413. doi:10.1016/j. biopsycho.2011.05.006

Kringelbach, M. L., \& Berridge, K. C. (2010). Towards a functional neuroanatomy of pleasure and happiness. Trends in Cognitive Science, 13(11), 479-487. doi: 10.1016/j.tics.2009.08.006

Ledrich, J., \& Gana, K. (2013). Relationship between attributional style, perceived control, self-esteem, and depressive mood in a nonclinical sample: A structural equation-modelling approach. Psychology and Psychotherapy: Theory, Research and Practice, 86(4), 413-430. doi:10.1111/j.2044-8341.2012.02067.x

Luck, S. J. (2014). An introduction to the event-related potential tecnique. MIT Press.

Luft, C. D. B. (2014). Learning from feedback: The neural mechanisms of feedback processing facilitating better performance. Behavioural Brain Research, 261, 356-368. doi:10.1016/j.bbr.2013.12.043 
Mikulincer, M. (1988). Reactance and helplessness following exposure to unsolvable problems: The effects of attributional style. Journal of Personality and Social Psychology, 54, 679-686. Retrieved from: https://www.ncbi.nlm.nih.gov/pubmed/3367284

Miller, W. R., \& Seligman, M. E. (1975). Depression and learned helplessness in man. Journal of Abnormal Psychology, 84(3), 228-238. doi: $10.1037 / \mathrm{h} 0076720$

Miller, A., \& Tomarken, A. J. (2001). Task-dependent changes in frontal brain asymmetry: Effects of incentive cues, outcome expectancies, and motor responses. Psychophysiology, 38(3), 500-511. doi:10. $1111 / 1469-8986.3830500$

Mineka, S., \& Hendersen, R. W. (1985). Controllability and predictability in acquired motivation. Annual Review of Psychology, 36, 495-529. doi:10.1146/annurev.psych.36.1.495

Mischel, W., \& Shoda, Y. (2010). The situated person. The Mind in Context, 149-173.

Nusslock, R., Shackman, A. J., Harmon-Jones, E., Alloy, L. B., Coan, J. A., \& Abramson, L. Y. (2011). Cognitive vulnerability and frontal brain asymmetry: Common predictors of first prospective depressive episode. Journal of Abnormal Psychology, 120(2), 497-503. doi:10.1037/a0022940

Nusslock, R., Walden, K., \& Harmon-Jones, E. (2015). Asymmetrical frontal cortical activity associated with differential risk for mood and anxiety disorder symptoms: An RDoC Perspective. International Journal of Psychophysiology: Official Journal of the International Organization of Psychophysiology. doi:10.1016/j. ijpsycho.2015.06.004

Petrantonakis, P. C., \& Hadjileontiadis, L. J. (2011). A novel emotion elicitation index using frontal brain asymmetry for enhanced EEGbased emotion recognition. IEEE Transactions on Information Technology in Biomedicine, 15(5), 737-746. doi:10.1109/TITB. 2011.2157933

Pfurtscheller, G. (2001). Functional brain imaging based on ERD / ERS. Vision Research, 41, 1257-1260. doi:10.1016/S0042-6989(00) 00235-2

Pornpattananangkul, N., \& Nusslock, R. (2016). Willing to wait: elevated reward-processing EEG activity associated with a greater preference for larger-but-delayed rewards. Neuropsychologia. doi:10.1016/j. neuropsychologia.2016.07.037

Salomons, T. V., Nusslock, R., Detloff, A., Johnstone, T., \& Davidson, R. J. (2015). Neural emotion regulation circuitry underlying anxiolytic effects of perceived control over pain. Journal of Cognitive Neuroscience, 27(2), 1-10. doi:10.1162/jocn

Sanjuán, P., \& Magallares, A. (2009). A longitudinal study of the negative explanatory style and attributions of uncontrollability as predictors of depressive symptoms. Personality and Individual Differences, 46(7), 714-718. doi:10.1016/j.paid.2009.01.030
Schultz, W. (2002). Getting formal with dopamine and reward. Neuron, 36(2), 241-263. doi:10.1016/S0896-6273(02)00967-4

Schweimer, J., \& Hauber, W. (2006). Dopamine D1 receptors in the anterior cingulate cortex regulate effort-based decision making. Learning \& Memory, 777-782. doi:10.1101/lm.409306.role

Semlitsch, H. V., Anderer, P., Schuster, P., \& Presslich, O. (1986). A solution for reliable and valid reduction of ocular artifacts, applied to the P300 ERP. Psychophysiology, 23(6), 695-703. doi:10.1111/j. 1469-8986.1986.tb00696.x

Sobotka, S. S., Davidson, R. J., \& Senulis, J. A. (1992). Anterior brain electrical asymmetries in response to reward and punishment. Electroencephalography and Clinical Neurophysiology, 83(4), 236-247. doi:10.1016/0013-4694(92)90117-Z

Thompson, S. C. (1981). Will it hurt less if I can control it? A complex answer to a simple question. Psychological Bulletin, 90(1), 89-101. doi: 10.1037/0033-2909.90.1.89

Tomarken, A., Davidson, R. J., Wheeler, R. E., \& Kinney, L. (1992). Psychometric properties of resting anterior EEG asymmetry: Temporal stability and internal consistency. Psychophysiology, 29(5), 576-592. doi:10.1111/j.1469-8986.1992.tb02034.x

Udo de Haes, J. I., Maguire, P. R., Jager, P. L., Paans, A. M. J., \& Den Boer, J. A. (2007). Methylphenidate-Induced activation of the anterior cingulate but not the striatum: A[15O]-H20 Pet Study in Healthy Volunteers. Human Brain Mapping, 28(7), 133-157. doi: 10.1002/hbm.20293

Wacker, J., Chavanon, M. L., \& Stemmler, G. (2006). Investigating the dopaminergic basis of extraversion in humans: A multilevel approach. Journal of Personality and Social Psychology, 91(1), 171187. doi:10.1037/0022-3514.91.1.171

Wacker, J., Chavanon, M. L., \& Stemmler, G. (2010). Resting EEG signatures of agentic extraversion: New results and meta-analytic integration. Journal of Research in Personality, 44(2), 167-179. doi:10.1016/j.jrp.2009.12.004

Wacker, J., Dillon, D. G., \& Pizzagalli, D. A. (2009). The role of the nucleus accumbens and rostral anterior cingulate cortex in anhedonia: Integration of resting EEG, fMRI, and volumetric techniques. NeuroImage, 46(1), 327-337. doi:10.1016/j.neuroimage.2009.01. 058.The

Wacker, J., \& Gatt, J. M. (2010). Resting posterior versus frontal delta/ theta EEG activity is associated with extraversion and the COMT VAL158MET polymorphism. Neuroscience Letters, 478(2), 88-92. doi:10.1016/j.neulet.2010.04.071

Walden, K., Pornpattananangkul, N., Curlee, A., McAdams, D. P., \& Nusslock, R. (2015). Posterior versus frontal theta activity indexes approach motivation during affective autobiographical memories. Cognitive Affective Behavioral Neuroscience, 15(1), 132-144. doi: 10.3758/s13415-014-0322-7 